\title{
Intrinsic viscosities of non-spherical colloids by Brownian dynamics simulations
}

Cite as: J. Chem. Phys. 151, 184902 (2019); https://doi.org/10.1063/1.5127001

Submitted: 07 September 2019 . Accepted: 27 October 2019 . Published Online: 14 November 2019

Duraivelan Palanisamy (D), and Wouter K. den Otter (D)

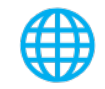

View Online
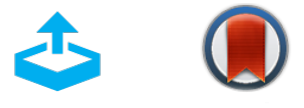

\section{ARTICLES YOU MAY BE INTERESTED IN}

Hydrodynamic correlations of viscoelastic fluids by multiparticle collision dynamics simulations

The Journal of Chemical Physics 151, 194110 (2019); https://doi.org/10.1063/1.5126082

Chemical Physics of Active Matter

The Journal of Chemical Physics 151, 114901 (2019); https://doi.org/10.1063/1.5125902

Thermal versus mechanical unfolding in a model protein

The Journal of Chemical Physics 151, 185105 (2019); https://doi.org/10.1063/1.5126071

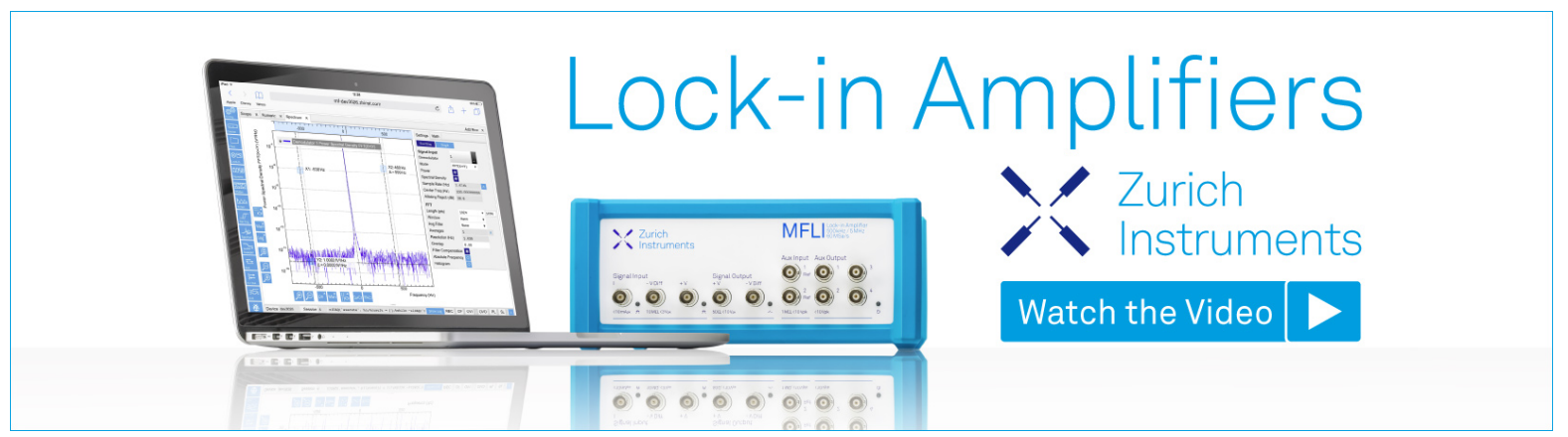




\title{
Intrinsic viscosities of non-spherical colloids by Brownian dynamics simulations
}

\author{
Cite as: J. Chem. Phys. 151, 184902 (2019); doi: 10.1063/1.5127001 \\ Submitted: 7 September 2019 - Accepted: 27 October 2019 • \\ Published Online: 13 November 2019
}

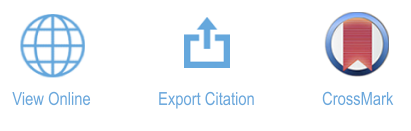

Duraivelan Palanisamy (D) and Wouter K. den Otter ${ }^{a)}$ (D)

\begin{abstract}
AFFILIATIONS
Multi-Scale Mechanics, Faculty of Engineering Technology and MESA+ Institute for Nanotechnology, University of Twente, P.O. Box 217, 7500 AE Enschede, The Netherlands
\end{abstract}

a) Electronic mail: w.k.denotter@utwente.nl

\begin{abstract}
A numerical study is presented on the intrinsic viscosities of sheared dilute suspensions of nonspherical Brownian colloidal particles. The simulations confirm theoretical predictions on the intrinsic viscosities of highly oblate and highly prolate spheroids in the limits of weak and strong Brownian noise (i.e., for low and high Péclet numbers). Numerical data and fit functions are provided covering the entire shearthinning regime, for spheroids ranging from highly oblate to highly prolate. The tumbling motion and intrinsic viscosities of a hemispherical cap and a helix are briefly discussed.
\end{abstract}

Published under license by AIP Publishing. https://doi.org/10.1063/1.5127001

\section{INTRODUCTION}

In his thesis, submitted in the annus mirabilis 1905, Einstein showed that the viscosity of a dilute suspension of spherical colloids increases proportionally with the colloidal volume fraction at a low Reynolds number. ${ }^{1,2}$ The extension of this result to nonspherical particles has attracted many researchers ever since. Jeffery analytically solved the periodic tumbling motion of spheroids-ellipsoidal particles with a rotational symmetry axis-in a simple shear flow, in the absence of Brownian motion. ${ }^{3}$ This tumbling motion is common to particles of almost all shapes. ${ }^{4,5}$ Jeffery also determined the variation of the shear stress with the orientation of the particle, which led him to speculate that the steady-state orientation distribution corresponds with the minimum rate of energy dissipation. ${ }^{3}$ Because the Jeffery orbits are closed, the viscosity of a dilute solution of noninteracting particles is determined by the initial orientation of these particles. This peculiar dependence is broken, and memory of the initial distribution is lost, by deviations from these orbits induced by inertial effects and/or Brownian motion. ${ }^{6}$ The combination of shear flow with Brownian motion involves constructing and solving a Fokker-Planck equation for the orientation distribution function, followed by an evaluation of the orientation-averaged stress. Two routes are taken in the literature, depending on the rotary Péclet number Pe, i.e., the ratio of the shear rate to the rotational diffusion coefficient. For low Pe, Burgers treated shear as a perturbation to the Brownian rotational motion, with the latter giving rise to a uniform distribution on the unit sphere. Closely related expressions for the intrinsic viscosities of highly prolate and/or oblate spheroids were derived by Onsager, ${ }^{8}$ Kuhn and Kuhn, ${ }^{9}$ Giesekus, ${ }^{10}$ and Hinch and Leal, ${ }^{11}$ with Simha ${ }^{12}$ obtaining an expression covering all aspect ratios. For high Pe, Leal and Hinch treated the Brownian motion as a perturbation to the Jeffery orbit ${ }^{11,13}$ giving rise to a slow diffusion of the orbital coordinate, i.e., the constant of the motion in Jeffery's solution that determines the shape of the orbit. To the best of our knowledge, there are no analytic solutions for the intermediate regime, for Pe of the order of unity, where the shear-induced motion and Brownian motion are of comparable importance.

Experimental measurements on the intrinsic viscosities of suspensions of Brownian particles show a surprising variation between different researcher groups, with values for supposedly identical systems varying by as much as an order of magnitude. ${ }^{14-18}$ This indicates that the experiments are very sensitive to the prevailing conditions, including the Reynolds number, polydispersity of the colloids, interactions between the colloids, and interactions between the colloids and the suspending fluid. For rods and ellipsoidal 
colloids, the quadratic scaling behavior of the intrinsic viscosity with the aspect ratio is clearly borne out by the experimental data, ${ }^{16}$ while it is difficult to confirm the theoretically derived proportionality factor.

Computer simulations provide an ideal testing ground for exploring theoretical models. The motion of spheroidal and ellipsoidal colloids in shear flows is attracting attention lately, with authors studying the impact of particle inertia, ${ }^{19,20}$ of fluid inertia, ${ }^{2}$ of both inertias, ${ }^{2-25}$ of weak Brownian noise, ${ }^{26}$ and of the combination thereof. ${ }^{27}$ The hydrodynamic contributions to the motion are accounted for by the explicit solution of time-varying flow and stress fields, or by analytic approximation in the form of mobility and resistance matrices. Similar approaches have been applied to more complex rigid bodies, including two-bladed paddles, ${ }^{28}$ fractals of sticky particles, ${ }^{29}$ curved nonchiral fibers, ${ }^{30}$ and irregular particles with edges and holes. ${ }^{31}$ The emphasis in most of these studies is on the dynamics of the particles, e.g., the phase behavior and chaos of the tumbling orbits ${ }^{20,22,32,33}$ or the segregation of chiral particles in a shear flow, ${ }^{34}$ while some studies also explored the resulting intrinsic viscosities of dilute suspensions of these particles. ${ }^{17,24,26,35,36}$ Freely available packages like BEST ${ }^{37}$ and HYDRO $++^{38}$ readily calculate the mobility matrices of arbitrarily shaped colloids and their intrinsic viscosities at zero shear rate. $^{39,40}$

Our objective is to explore the intrinsic viscosity, in particular, the interplay between shear flow and Brownian motion that gives rise to shear thinning for elongated colloids. We have recently shown that Brownian Dynamics (BD) simulations of isolated rigid bodies benefit greatly from describing the rotational motions in terms of unit-quaternions, i.e., a four-vector of unit length that enables expressing all elements of a rotation matrix in quadratic forms. ${ }^{41,42}$ Several authors have exploited quaternions in BD simulations of colloids to avoid the divergencies occurring when using three rotational coordinates, like the Euler angles or the Cartesian components of the rotation vector. ${ }^{26,36,43-45}$ Less appreciated is that quaternions have the additional advantages of eliminating both a metric tensor term, associated with BD simulations in nonCartesian coordinates, and a mobility term, associated with the orientation dependence of the mobility matrix. ${ }^{42,46}$ The latter cancellation occurs only if the orientation of the colloid is determined relative to the hydrodynamic center in the mobility picture. ${ }^{42}$ This rotational $\mathrm{BD}$ scheme is briefly described in Sec. II, along with the procedure to extract shear stresses from the simulations. Both are used in Sec. III to calculate the intrinsic viscosities of dilute suspensions of spheroids. The algorithm is validated against the aforementioned theoretical predictions of the intrinsic viscosities of spheroids at low and high Péclet numbers. Numerical data and fit functions are provided covering the entire intermediate shearthinning region for aspect ratios ranging from $1 / 100$ to 100 . Simulations are also presented of two less symmetric bodies, namely, a spherical cap and a helix. The former performs a more intricate Jeffery orbit, combining the tumbling motion of a spheroid with a periodic orbit of its hydrodynamic center that has been solved analytically. ${ }^{47}$ The latter achiral particle is of interest because it is known to drift along the vorticity direction, a feature with potential application in the separation of left- and right-handed colloids and enantiomeric molecules. ${ }^{34,48-52}$ The main conclusions are summarized in Sec. IV.

\section{THEORY}

\section{A. Brownian dynamics}

Consider a colloidal particle experiencing a force $\overline{\mathbf{f}}$ and a torque $\overline{\boldsymbol{\tau}}$, both derived from a potential $\Phi$, while suspended in a Newtonian fluid in a linear flow field,

$$
\overline{\mathbf{v}}_{\infty}(\overline{\mathbf{r}})=\overline{\mathbf{v}}_{\infty}^{0}+\overline{\boldsymbol{\omega}}_{\infty} \times \overline{\mathbf{r}}+\overline{\overline{\mathbf{E}}}_{\infty} \overline{\mathbf{r}}
$$

where $\overline{\mathbf{r}}$ denotes the position, $\overline{\mathbf{v}}_{\infty}^{0}$ denotes the flow velocity at the origin of the laboratory coordinate system, and the angular velocity $\overline{\boldsymbol{\omega}}_{\infty}$ and the strain rate $\overline{\overline{\mathbf{E}}}_{\infty}$ are constant throughout the flow. In the Stokes approximation, the velocity $\overline{\mathbf{v}}$ and angular velocity $\overline{\boldsymbol{\omega}}$ of a colloid at position $\overline{\mathbf{x}}$ are solved from ${ }^{5}$

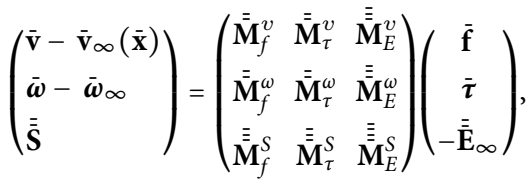

where $\overline{\overline{\mathbf{S}}}$ is the stress exerted by the particle on the fluid, i.e., the symmetric first moments of the deviatoric stress field $\overline{\overline{\boldsymbol{\sigma}}}(\overline{\mathbf{r}})$ over the surface of the particle, ${ }^{53-55}$ with unit Newton meter. The velocity differences between the colloid and the flow result from balances between the potential force and torque driving the particle and the solvent friction-represented by the generalized mobility matrix $\mathbf{M}$-opposing the relative motion. The stress is obtained by applying Newton's third law to the hydrodynamic stresslet acting on a moving colloid in a flow field. The generalized mobility matrices of spherical and ellipsoidal bodies are tabulated in the literature, ${ }^{54}$ while those of more generally shaped rigid bodies can be constructed either by the boundary element method, ${ }^{28,56,57}$ as in BEST, ${ }^{37}$ or by assuming the body composed of spherical primary particles, ${ }^{30,42,44,58-60}$ as in HYDRO++. ${ }^{38}$ The latter approach is followed here for nonspheroidal colloids: Appendix A provides a brief summary of the method, freely available as Oseen $11{ }^{61}$ Two brief comments on the notation: the bars in the above expression highlight that the generalized mobility matrix combines matrices of various ranks; the bars will be omitted for convenience henceforth. The nine blocks of the mobility matrix are labelled with two indices, with the lower index specifying the multiplication partner and the top index highlighting the ensuing result.

For a rigid body composed of multiple beads, the position of the $i$ th bead in the space frame (s) is related to that in the body frame (b) by

$$
\mathbf{x}_{i}^{(\mathrm{s})}=\mathbf{x}^{(\mathrm{s})}+\mathbf{A}_{(\mathrm{b})}^{(\mathrm{s})} \mathbf{x}_{i}^{(\mathrm{b})},
$$

with $\mathbf{A}_{(\mathrm{b})}^{(\mathrm{s})}$ the rotation matrix from the body to the space frame and $\mathbf{x}^{(\mathrm{s})}$ the reference position of the colloid, which coincides with the origin of the body-based coordinate system. The generalized mobility matrix of the colloid is constant in the body-based frame and rotates with the body in the space frame, e.g.,

$$
\begin{aligned}
M_{f(\mathrm{~s}) \gamma}^{v(\mathrm{~s}) \alpha} & =A_{(\mathrm{b}) \kappa}^{(\mathrm{s}) \alpha} M_{f(\mathrm{~b}) \mu}^{v(\mathrm{~b}) \kappa} A_{(\mathrm{s}) \gamma}^{(\mathrm{b}) \mu}, \\
M_{\tau(\mathrm{s}) \gamma}^{S(\mathrm{~s}) \gamma} & =A_{(\mathrm{b}) \kappa}^{(\mathrm{s}) \alpha} A_{(\mathrm{b}) \lambda}^{(\mathrm{s}) \beta} M_{\tau(\mathrm{b}) \mu}^{S(\mathrm{~b})} A_{(\mathrm{s}) \gamma}^{(\mathrm{b}) \mu}, \\
M_{E(\mathrm{~s}) \gamma \delta}^{S(\mathrm{~s}) \alpha \beta} & =A_{(\mathrm{b}) \kappa}^{(\mathrm{s}) \alpha} A_{(\mathrm{b}) \lambda}^{(\mathrm{s}) \beta} M_{E(\mathrm{~b}) \mu \nu}^{S(\mathrm{~b}) \kappa \lambda} A_{(\mathrm{s}) \gamma}^{(\mathrm{b}) \mu} A_{(\mathrm{s}) \delta}^{(\mathrm{b}) \nu},
\end{aligned}
$$


where the Einstein convention of summing over repeated indices, appearing once up and once down, is implied. Hence, it suffices to perform one computationally intensive evaluation of the hydrodynamic problem to arrive at the mobility matrix in the body frame, which is then combined with cheap rotations to obtain the mobility matrix in the space frame for any orientation of the body.

A colloidal particle suspended in a quiescent fluid will perform a Brownian motion. In terms of generalized coordinates $\mathbf{Q}$, in this case, comprising the particle's position $\mathbf{x}^{(s)}$ and its orientation $\mathbf{q}$, the discretized equation of motion of a particle in a potential $\Phi$ reads as ${ }^{62-64}$

$$
\begin{aligned}
\Delta \mathbf{Q}(t) & =\mathbf{Q}(t+\Delta t)-\mathbf{Q}(t) \\
& =-\mathbf{M}_{F}^{\dot{Q}} \nabla_{Q}\left(\Phi-\frac{1}{2} k_{B} T \ln g_{Q}\right) \Delta t+k_{B} T \nabla_{Q} \cdot \mathbf{M}_{F}^{\dot{Q}} \Delta t+\delta \mathbf{Q},
\end{aligned}
$$

where $t$ denotes the time, $\Delta t$ the integration time step, $\mathbf{M}_{F}^{\dot{Q}}$ the mobility matrix converting generalized forces $\mathbf{F}=-\nabla_{Q} \Phi$ into generalized velocities $\dot{\mathbf{Q}}, k_{B}$ Boltzmann's constant, $T$ the temperature, $g_{Q}$ the metric of the coordinate space, and $\delta \mathbf{Q}$ the Brownian displacements. In this forward-Euler or Itô representation of a stochastic equation, all quantities in Eq. (5b) are evaluated at time $t$ using the coordinate values at the start of the step. Expressions for the metric have been derived for simulations based on Euler angles or the three Cartesian coordinates of a rotation vector. ${ }^{65-69}$ The Brownian displacements have zero mean and no memory of preceding steps (Markovian) while they are correlated with the fluctuationdissipation theorem, ${ }^{62-64}$

$$
\langle\delta \mathbf{Q} \otimes \delta \mathbf{Q}\rangle=2 k_{B} T \mathbf{M}_{F}^{\dot{Q}} \Delta t .
$$

A subtle correction is required when the mobility matrix is a function of the coordinates, in which case the Brownian motion acquires a bias accounted for by the divergence term in Eq. (5b). Note that mobility matrices are usually expressed in the form of Eq. (2), in terms of velocities and forces along and around the Cartesian axes, while $\mathbf{M}_{F}^{\dot{Q}}$ requires expression in terms of the generalized velocities and forces. The conversions of the Cartesian space-based $\mathbf{M}_{\tau(\mathrm{s})}^{v(\mathrm{~s})}$, $\mathbf{M}_{f(\mathrm{~s})}^{\omega(\mathrm{s})}$, and $\mathbf{M}_{\tau(\mathrm{s})}^{\omega(\mathrm{s})}$ to their counterparts in rotational velocities $\dot{\mathbf{q}}$ and corresponding forces $-\nabla_{q} \Phi$ are realized by multiplications with the matrix $\mathbf{B}_{\omega(\mathrm{s})}^{\dot{q}}=\partial \mathbf{q} / \partial \boldsymbol{\psi}^{(\mathrm{s})}=\partial \dot{\mathbf{q}} / \partial \boldsymbol{\omega}^{(\mathrm{s})}$ relating (the velocity of) changes in the orientation coordinates $\mathbf{q}$ to (the velocity of) infinitesimal rotations $\psi^{(s)}$ around the space-based Cartesian axes. This conversion matrix diverges for certain values of the Euler angles, while converging singularities are encountered when using a rotation vector. $^{65-69}$ These complications are avoided when describing rotations in terms of unit quaternions, ${ }^{41}$ i.e., a four-vector $\mathbf{q}$, at the relatively minor effort of a length constraint, $|\mathbf{q}|=1$. With this choice, the rotation matrix is quadratic and the transformation matrix is linear in the vector components, see Appendix B, while the gradient of the metric lies parallel to $\mathbf{q}$ and therefore cancels against the constraint. ${ }^{46}$ Furthermore, if the body-based mobility matrix is calculated relative to the hydrodynamic mobility center of the colloid, i.e., if the origin of the body-based coordinate system is chosen such that $\mathbf{M}_{\tau(\mathrm{b})}^{v(\mathrm{~b})}=\mathbf{M}_{f(\mathrm{~b})}^{\omega(\mathrm{b})}$, the divergence term vanishes identically from the equation of motion. ${ }^{42}$
By combining the above expressions, one arrives at the discretized equation of motion of a rigid Brownian colloid suspended in a flow and subject to a potential,

$$
\begin{aligned}
\left(\begin{array}{c}
\Delta \mathbf{x} \\
\Delta \mathbf{q}
\end{array}\right)= & \left(\begin{array}{cc}
\mathbf{A}_{(\mathrm{b})}^{(\mathrm{s})} & \mathbf{0} \\
\mathbf{0} & \mathbf{B}_{\omega(\mathrm{b})}^{\dot{q}}
\end{array}\right)\left[\left(\begin{array}{ccc}
\mathbf{M}_{f}^{v} & \mathbf{M}_{\tau}^{v} & \mathbf{M}_{E}^{v} \\
\mathbf{M}_{f}^{\omega} & \mathbf{M}_{\tau}^{\omega} & \mathbf{M}_{E}^{\omega}
\end{array}\right)_{(\mathrm{b})}^{(\mathrm{b})}\left(\begin{array}{c}
\mathbf{A}_{(\mathrm{s})}^{(\mathrm{b})} \mathbf{f}^{(\mathrm{s})} \\
\mathbf{A}_{(\mathrm{s})}^{(\mathrm{b})} \boldsymbol{\tau}^{(\mathrm{s})} \\
-\mathbf{A}_{(\mathrm{s})}^{(\mathrm{b})} \mathbf{E}_{\infty}^{(\mathrm{s})} \mathbf{A}_{(\mathrm{b})}^{(s)}
\end{array}\right) \Delta t\right. \\
& \left.+\left(\begin{array}{l}
\delta \mathbf{x}^{(\mathrm{b})} \\
\delta \boldsymbol{\psi}^{(\mathrm{b})}
\end{array}\right)\right]+\left(\begin{array}{r}
\mathbf{v}_{\infty}^{(\mathrm{s})} \\
\mathbf{B}_{\omega(\mathrm{s})}^{\dot{q}} \boldsymbol{\omega}_{\infty}^{(\mathrm{s})}
\end{array}\right) \Delta t+\left(\begin{array}{c}
\mathbf{0} \\
\lambda \mathbf{q}
\end{array}\right),
\end{aligned}
$$

with $\Delta \mathbf{x}$ and $\Delta \mathbf{q}$ the increments of the position and orientation, respectively, over a time step $\Delta t$. In the first term between the square brackets, the force, torque, and strain in the space frame are converted to their counterparts in the body frame by a rotation, followed by application of Eq. (2) in the body frame [where for compactness of notation, the sub- and superscripts (b) are appended to the entire matrix rather than to every block], thereby arriving at linear and angular velocities in the body frame; the matrix preceding the square brackets converts the linear velocities back to the space frame and the angular velocities to quaternion velocities. Expressions for the rotation matrices $\mathbf{A}_{(\mathrm{b})}^{(\mathrm{s})}$ and $\mathbf{A}_{(\mathrm{s})}^{(\mathrm{b})}$, as well as for the conversion matrices $\mathbf{B}_{\omega(\mathrm{b})}^{\dot{q}}$ and $\mathbf{B}_{\omega(\mathrm{s})}^{\dot{q}}$, are provided in Appendix B. The penultimate term in the equation of motion describes displacements due to the flow field. In the second term between the square brackets, the random displacements $\delta \mathbf{x}^{(\mathrm{b})}$ and $\delta \psi^{(\mathrm{b})}$ in the body-frame, which obey a fluctuation-dissipation theorem akin to Eq. (6), are sampled using

$$
\left(\begin{array}{l}
\delta \mathbf{x}^{(\mathrm{b})} \\
\delta \psi^{(\mathrm{b})}
\end{array}\right)=\sqrt{2 k_{B} T \Delta t}\left[\left(\begin{array}{ll}
\mathbf{M}_{f}^{v} & \mathbf{M}_{\tau}^{v} \\
\mathbf{M}_{f}^{\omega} & \mathbf{M}_{\tau}^{\omega}
\end{array}\right)_{(\mathrm{b})}^{(\mathrm{b})}\right]^{1 / 2} \boldsymbol{\Theta}_{x \psi},
$$

where the square root of a symmetric matrix is again a symmetric matrix, and $\Theta_{x \psi}$ represents a vector of six uncorrelated random numbers without memory, each of zero mean and unit average. Because the columns of $\mathbf{B}_{\omega(\mathrm{b})}^{\dot{q}}$ and $\mathbf{B}_{\omega(\mathrm{s})}^{\dot{q}}$ are all perpendicular to $\mathbf{q}$, the length of the quaternion vector is conserved in the limit of infinitely small time steps. To conserve the length in a finite time step, a constraint with an undetermined Lagrange multiplier $\lambda$ is included in the last term to the equation of motion. Using $\mathbf{q}^{u}$ to denote the quaternion vector after the unconstrained step, as obtained by assuming $\lambda=0$, the required value of $\lambda$ follows from the constraint condition,

$$
|\mathbf{q}(t+\Delta t)|=\left|\mathbf{q}^{u}(t+\Delta t)+\lambda \mathbf{q}(t)\right|=1,
$$

which readily yields a quadratic equation in $\lambda$.

\section{B. Stress and viscosity}

The stress in the fluid is derived from Eq. (2), whose bottom line provides an expression for minus the instantaneous hydrodynamic stress on a non-Brownian colloid subjected to a potential and flow. To include the Brownian contribution, the Brownian displacements in the discretized equation of motion are converted to Brownian forces and torques, to be added to the 
potential forces and torques in the stress expression. The stress then reads as

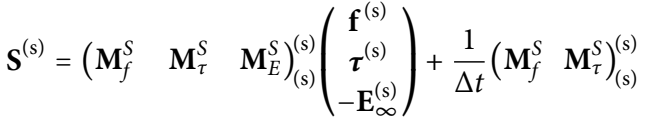

$$
\begin{aligned}
& \times\left[\left(\begin{array}{ll}
\mathbf{M}_{f}^{v} & \mathbf{M}_{\tau}^{v} \\
\mathbf{M}_{f}^{\omega} & \mathbf{M}_{\tau}^{\omega}
\end{array}\right)_{(\mathrm{s})}^{(\mathrm{s})}\right]^{-1}\left(\begin{array}{c}
\delta \mathbf{x}^{(\mathrm{s})} \\
\delta \boldsymbol{\psi}^{(\mathrm{s})}
\end{array}\right)+k_{B} T \mathbf{G}^{(\mathrm{s})},
\end{aligned}
$$

where $\delta \mathbf{x}^{(s)}$ and $\delta \psi^{(s)}$ denote the space-based representation of the Brownian displacements in Eq. (8). While the contribution of the Brownian displacements to the stress clearly averages out to zero, the Brownian motion also gives rise to the subtle nonvanishing last term in the above expression; the reader is referred to the literature for derivations of this term ${ }^{35,70,71}$ (see also Appendix A),

$$
\mathbf{G}^{(s)}=\left(\begin{array}{c}
\nabla \\
\mathcal{R}
\end{array}\right) \cdot\left(\begin{array}{ll}
\mathbf{M}_{f}^{S} & \mathbf{M}_{\tau}^{S}
\end{array}\right)_{(\mathrm{s})}^{(\mathrm{s})}=\mathcal{R} \cdot \mathbf{M}_{\tau(\mathrm{s})}^{S(\mathrm{~s})}
$$

where $\mathcal{R}$ is the rotational operator ${ }^{72,73}$ and the last step follows from the translational invariance of the space-based mobility matrix of an isolated colloid. With $\mathbf{M}_{\tau(\mathrm{s})}^{S(\mathrm{~s})}$ expressed in terms of $\mathbf{M}_{\tau(\mathrm{b})}^{S(\mathrm{~b})}$ and $\mathbf{A}_{\text {(b) }}^{(\mathrm{s})}$ by Eq. (4b) and using the relations derived in Appendix C, the divergence is evaluated as

$$
\begin{aligned}
\mathbf{G}^{(\mathrm{s})} & =\mathbf{A}_{(\mathrm{b})}^{(\mathrm{s})} \mathbf{G}^{(\mathrm{b})} \mathbf{A}_{(\mathrm{s})}^{(\mathrm{b})}, \\
G^{(\mathrm{b}) \kappa \lambda} & =\epsilon_{\rho}^{\kappa \pi} M_{\tau(\mathrm{b}) \pi}^{S(\mathrm{~b}) \rho \lambda}+\epsilon_{\rho}^{\lambda \pi} M_{\tau(\mathrm{b}) \pi}^{S(\mathrm{~b}) \kappa \rho},
\end{aligned}
$$

where $\mathbf{G}^{(\mathrm{b})}$ is constant in the body frame; the Levi-Civita symbol $\epsilon_{\gamma}^{\alpha \beta}$ equals $+1(-1)$ when $\alpha, \beta, \gamma$ is a cyclic (anticyclic) permutation of $\{x, y, z\}$ and equals zero otherwise. Combining the above steps, the stress is conveniently calculated in the body frame as

$$
\begin{aligned}
& \mathbf{S}^{(\mathrm{b})}=\left(\begin{array}{lll}
\mathbf{M}_{f}^{S} & \mathbf{M}_{\tau}^{S} & \mathbf{M}_{E}^{S}
\end{array}\right)_{(\mathrm{b})}^{(\mathrm{b})}\left(\begin{array}{c}
\mathbf{A}_{(\mathrm{s})}^{(\mathrm{b})} \mathbf{f}^{(\mathrm{s})} \\
\mathbf{A}_{(\mathrm{s})}^{(\mathrm{b})} \boldsymbol{\tau}^{(\mathrm{s})} \\
-\mathbf{A}_{(\mathrm{s})}^{(\mathrm{b})} \mathbf{E}_{\infty}^{(\mathrm{s})} \mathbf{A}_{(\mathrm{b})}^{(\mathrm{s})}
\end{array}\right) \\
& +\frac{1}{\Delta t}\left(\begin{array}{ll}
\mathbf{M}_{f}^{S} & \mathbf{M}_{\tau}^{S}
\end{array}\right)_{(\mathrm{b})}^{(\mathrm{b})}\left[\left(\begin{array}{ll}
\mathbf{M}_{f}^{v} & \mathbf{M}_{\tau}^{v} \\
\mathbf{M}_{f}^{\omega} & \mathbf{M}_{\tau}^{\omega}
\end{array}\right)_{(\mathrm{b})}^{(\mathrm{b})}\right]^{-1}\left(\begin{array}{l}
\delta \mathbf{x}^{(\mathrm{b})} \\
\delta \boldsymbol{\psi}^{(\mathrm{b})}
\end{array}\right)+k_{B} T \mathbf{G}^{(\mathrm{b})},
\end{aligned}
$$

where the Brownian displacements are identical to those in the equation of motion, see Eq. (7), followed by the transformation,

$$
\mathbf{S}^{(\mathrm{s})}=\mathbf{A}_{(\mathrm{b})}^{(\mathrm{s})} \mathbf{S}^{(\mathrm{b})} \mathbf{A}_{(\mathrm{s})}^{(\mathrm{b})},
$$

to the space frame.

The presence of colloids in the fluid affects the velocity and stress fields in the fluid and thereby raises the viscosity of the pure fluid, $\eta_{0}$, to the effective viscosity of the suspension, $\eta_{\mathrm{s}}$. A convenient way to express this impact is the intrinsic viscosity $[\eta]$, defined by

$$
\eta_{\mathrm{s}}=\eta_{0}(1+[\eta] \phi)
$$

where $\phi$ denotes the (small) volume fraction of particles. For a dilute suspension of rigid spherical colloids, Einstein derived the seminal result $[\eta]=5 / 2 \cdot{ }^{1,2,55}$ By using that the viscosity is related to the average shear stress, one readily shows that

$$
[\eta]=\frac{1}{\eta_{0} v_{c}} \frac{\left\langle S_{x y}^{(s)}\right\rangle}{2 E_{x y}^{\infty(s)}},
$$

where $v_{c}$ denotes the volume of the colloid and $2 E_{x y}^{\infty}(s)=\dot{\gamma}$ for the simple shear flow adopted throughout this study, $\mathbf{v}_{\infty}(\mathbf{r})=j r^{y} \hat{\mathbf{e}}_{x}$ with $\dot{\gamma}$ the shear rate and $\hat{\mathbf{e}}_{x}$ the basis vector along the $x$ axis.

\section{Spheroids}

For a non-Brownian spheroid in a simple shear flow, with length $L$ along the axis of revolution, diameter $D$ along the two perpendicular axes, and aspect ratio $p=L / D$ (see Fig. 1), Jeffery solved the orbit traced out by the axis of revolution as a function of time $t^{3}$

$$
\begin{aligned}
\tan \theta & =C\left(\cos ^{2} \chi+p^{2} \sin ^{2} \chi\right)^{1 / 2}, \\
\tan \varphi & =p \tan \chi \\
\chi & =\kappa t=\frac{\dot{\gamma}}{p+(1 / p)} t,
\end{aligned}
$$

where $\theta$ denotes the angle of the symmetry axis relative to the vorticity direction and $\varphi$ the rotation angle around the vorticity axes (see Fig. 2), the revolution period $\mathcal{T}=2 \pi / \kappa$ is equal for all orbits, and $C$ is a constant of motion characterizing the orbit. A spheroid at $C=\infty$ performs a "tumbling" motion with its symmetry axis permanently perpendicular to the vorticity, in the $\theta=\pi / 2$ plane,

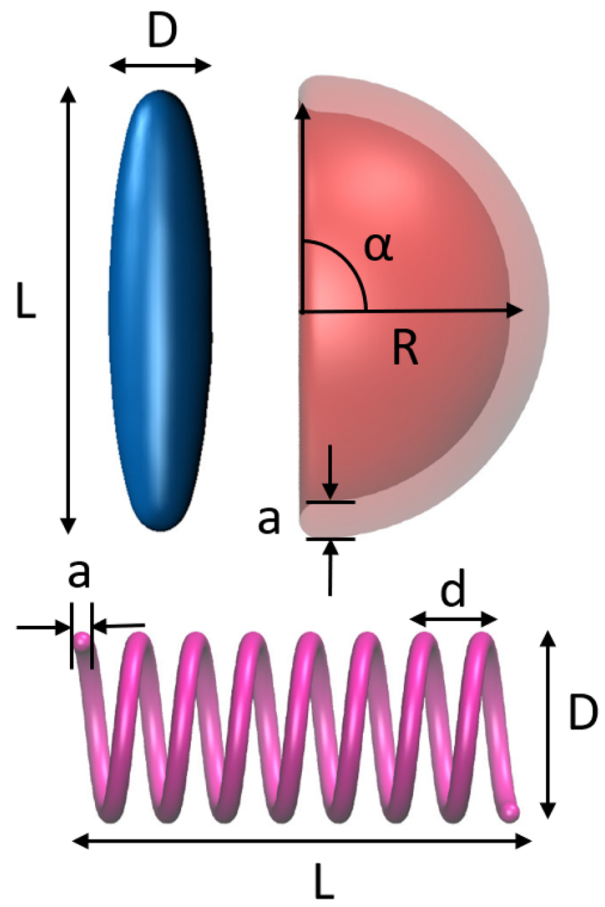

FIG. 1. Pictorial representations of the simulated bodies: a prolate spheroid (blue) of length $L$ and diameter $D$, a hemispherical cap (red) of radius $R$, thickness $a$, and top angle $\alpha$, and a helix (pink) of length $L$, diameter $D$, pitch $d$, and thickness $a$. 


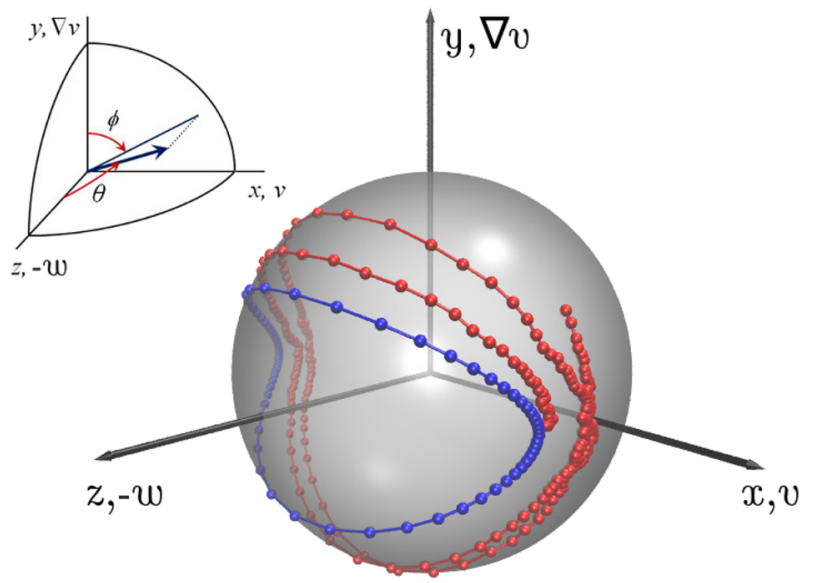

FIG. 2. Orientation of the linear shear flow field relative to the coordinate system, with the flow velocity $\mathbf{v}_{\infty}$, the velocity gradient $\nabla \mathbf{v}_{\infty}$, and the vorticity $\mathbf{w}$ parallel to the $x$-axis, the $y$-axis, and the (negative) $z$-axis, respectively. Also shown are the paths traced on the unit sphere (grey) by the symmetry axis of a nonBrownian (blue, $\mathrm{Pe}=\infty$ ) and a moderately Brownian (red, $\mathrm{Pe}=3 \cdot 10^{3}$ ) prolate $p=5$ spheroid suspended in this shear flow, with the dots denoting orientations at regular intervals in time. The symmetry axis moves at its slowest, and hence, the impact of the Brownian motion is at its largest, when the axis is nearly flow-aligned.

with a variable angular velocity $\dot{\varphi}$ that reaches minima (maxima) when the symmetry axis is parallel (perpendicular) to the flow direction. A spheroid at $C=0$ has its symmetry axis forever oriented along the vorticity direction, $\theta=0$, while "log-rolling" around this axis at a constant angular velocity. Intermediate values of $C$ correspond to a "kayaking" motion of the symmetry axis (see Fig. 2) with the spheroid simultaneously rotating around its symmetry axis (not shown). Jeffery also derived expressions for the instantaneous orientation-dependent intrinsic viscosity of a spheroid, based on the excess work required in shearing a particle-laden fluid vs the pure fluid. ${ }^{3}$ For instance, the intrinsic viscosity due to a particle tumbling at constant $\theta=\pi / 2$ varies with the tumbling angle $\varphi$ following

$$
[\eta]=\frac{4}{3 v_{\mathrm{c}}}\left(F \sin ^{2} 2 \varphi+G\right)
$$

where $F$ and $G$ are functions of the aspect ratio.

In the presence of Brownian noise, the motion is no longer deterministic and the orbit parameter $C$ is not conserved. For weak Brownian noise, $\mathrm{Pe} \rightarrow \infty$; Leal and Hinch ${ }^{11,13}$ assumed that the motion can be described by augmenting Jeffery's solution with a slow (compared to the tumbling period) diffusion of the orbit parameter $C$. The steady state orientation probability distribution then takes the form of a product,

$$
P(C, \tilde{\chi})=P_{C}(C) P_{\chi}(\tilde{\chi} \mid C),
$$

where $P_{\chi}(\tilde{\chi} \mid C)$, the conditional distribution of $\chi$ mapped to its modulo $\tilde{\chi}$ in the $(0, \pi)$ interval, is approximated by the distribution of $\tilde{\chi}$ for an unperturbed Jeffery orbit at the given $C$. The steady state distribution across these orbits, $P_{C}(C)$, is solved from a Fokker-Planck equation by demanding that the average flux crossing any closed Jeffery orbit equals zero. In combination with Jeffery's expression for the excess work, the intrinsic viscosity is obtained as

$$
[\eta]_{\infty}=\left\{\begin{array}{ccc}
3.183-1.792 p & \text { for } & p \rightarrow 0 \\
2+\frac{0.315 p}{\ln (2 p)-1.5} & \text { for } & p \rightarrow \infty
\end{array}\right.
$$

with the limiting behavior, ${ }^{11}$

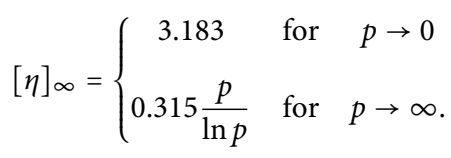

Since the numerical values in the published equations ${ }^{11,13}$ are inconsistent ( 3.183 vs 3.13 and 0.312 vs 0.315 ), we took the liberty of selecting those values that agree best with the simulation results presented below.

In the opposite limit of a strong Brownian noise, $\mathrm{Pe} \ll 1$, the orientation distribution in the angles $\theta$ and $\varphi$ was assumed by Burgers ${ }^{7}$ to be nearly isotropic, with the flow giving rise to a weak perturbation. Onsager, ${ }^{8}$ Simha, ${ }^{12}$ Kuhn and Kuhn, ${ }^{9}$ Giesekus, ${ }^{10}$ and Hinch and Leal ${ }^{11}$ derived identical limiting expressions for the intrinsic viscosity at $\mathrm{Pe} \rightarrow 0$,

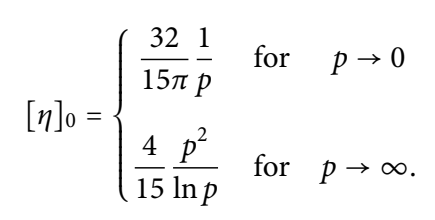

A theory for $[\eta]_{0}$ covering the entire range of $p$ was derived by Simha. ${ }^{12}$ To the best of our knowledge, there are no analytic solutions for intermediate Brownian noise, i.e., for the shear-thinning transition between these two extremes.

\section{RESULTS}

The equations of motion reviewed in Secs. II A and II B are now applied to spheroids in Sec. III A and to two more complex bodiesa hemispherical cap and helices (see Fig. 1) -in Sec. III B. The three independent units used in the simulations are $\epsilon$ for the energy, $\sigma$ for distance, and $\tau$ for time; all relevant quantities are expressible in these units. The imposed flow field is a simple shear, $\mathbf{v}_{\infty}(\mathbf{r})=\dot{\gamma} r^{y} \hat{\mathbf{e}}_{x}$ (see Fig. 2), with a constant shear rate $\dot{\gamma}=0.01 \tau^{-1}$. The solvent viscosity is fixed at $\eta_{0}=(6 \pi)^{-1} \epsilon \tau / \sigma^{3}$, and the time step is $\Delta t=0.01 \tau$. For computational reasons, the Péclet number-defined as $\mathrm{Pe}=\dot{\gamma} / D_{r}$ with the rotational diffusion coefficient $D_{r}$-is varied by changing the thermal energy, $k_{B} T$, and hence the strength of the Brownian noise. For ease of comparison with experiments and theory, the main results will be presented in terms of dimensionless numbers, e.g., the intrinsic viscosity, Péclet number, and aspect ratio.

\section{A. Spheroids}

Spheroidal particles provide an ideal testing ground for methodological development; the exact expression for the generalized mobility matrix ${ }^{54}$ is used in the $\mathrm{BD}$ simulations, and the 


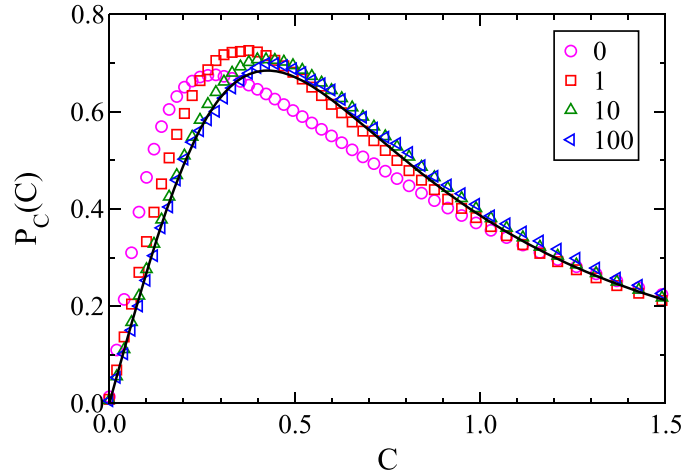

FIG. 3. Probability distributions of the Jeffery orbit parameter $C$ for prolate ellipsoids, $p=5$, in simple shear flows at various Péclet numbers (see the legend) and in a quiescent fluid $(\mathrm{Pe}=0)$. The solid line shows the theoretical prediction by Leal and Hinch for high Pe.

extracted intrinsic viscosities can be compared against theoretical solutions of $[\eta]_{0}$ and $[\eta]_{\infty}$.

\section{Distributions}

A typical path traced by the long axis of a prolate spheroid at high Pe is shown in Fig. 2, where the kayaking motion of the particle is still recognizable while the superimposed diffusion of the orbit parameter results in a nonclosed path. Probability distributions of the orbit parameter $C$ over long simulations are presented in Fig. 3 for a wide range of $\mathrm{Pe}=\dot{\gamma} / D_{r}$, where $D_{r}$ is obtained as $k_{B} T$ times the degenerate eigenvalue of $\mathbf{M}_{\tau}^{\omega}$. The sampled distribution at large Pe closely approximates the theoretical expression for $P_{C}(C)$ derived by Leal and Hinch ${ }^{13}$ in this limit. Note, however, that the distribution is fairly insensitive to the Péclet number. The distribution of the polar angles of the long axis reveals a much more pronounced impact of the Péclet number on orientation; see Fig. 4.

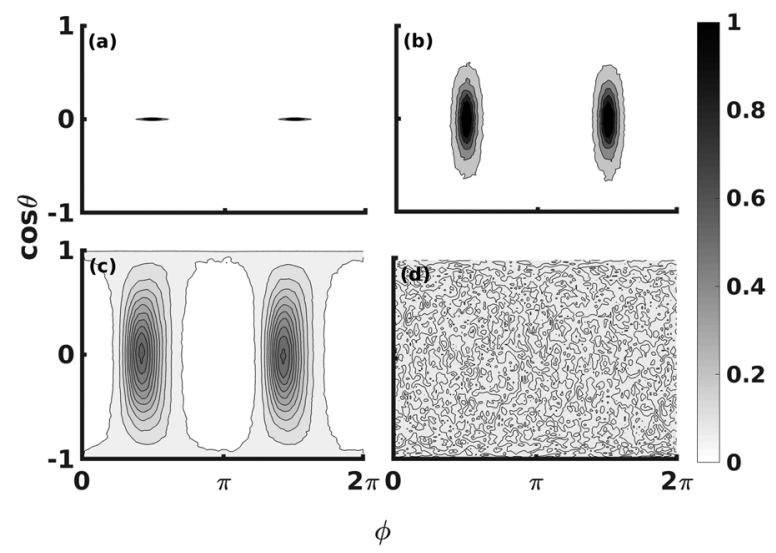

FIG. 4. Distributions of the orientation of the long axis of a prolate spheroid, $p=5$, (a) in a sheared fluid without thermal noise, $\mathrm{Pe}=\infty$, perpetually tumbling in a Jeffery orbit at $\theta=\pi / 2$, in shear flows with Brownian fluctuations at (b) $P e=3000$ and $(c) P e=30$, and (d) in a quiescent fluid with thermal noise, $P e=0$. The selected mapping turns an isotropic distribution into a uniform $P(\cos \theta, \varphi)=1 /(4 \pi)$.
In a quiescent fluid, the spheroid samples an isotropic probability distribution, as expected for a Brownian particle with no preferred orientation, while a clear preference for the two flow-aligned orientations, $\cos \theta=0$ (i.e., $\theta=\pi / 2$ ) and $\varphi=\{\pi / 2,3 \pi / 2\}$, emerges with increasing Pe. The peaks shift and sharpen with $\mathrm{Pe}$, as can be quantified by fitting the sampled distributions with bivariate normal distributions,

$$
P(\cos \theta, \varphi)=a+b_{1} e^{-c_{1} \cos ^{2} \theta-d_{1}\left(\varphi-\varphi_{1}\right)^{2}}+b_{2} e^{-c_{2} \cos ^{2} \theta-d_{2}\left(\varphi-\varphi_{2}\right)^{2}} .
$$

The locations of the peaks are plotted in Fig. 5 as a function of the Péclet number, for spheroids with aspect ratios of 5 and 20. At low Péclet, the particles are seen to preferably orient along the direction of the elongation component of the flow field, at $\varphi=\pi / 4$, while at high Péclet, the particles align along the flow field itself, at $\varphi=\pi / 2$, in accordance with the predictions by Burgers. The widths of the peaks, as represented by the standard deviations $\sigma_{\varphi}=\left(2 d_{i}\right)^{-1 / 2}$, are indicated in the figure by error bars. One clearly sees a narrowing of the distribution with increasing Péclet number. The good agreement between the corresponding fit parameters of the two peaks to $P$ indicates that the distributions are well-sampled (data not shown).

The simulations also permit an assessment of the impact of the Brownian motion on the tumbling period. In runs over a range of Péclet numbers, the increments and decrements of the angle $\varphi$ were added up to count the numbers of revolutions during the simulations. For ease of interpretation, the angle $\theta$ was constrained to the value of $\pi / 2$. In Fig. 6 , the average tumbling periods are compared against the periods calculated by Jeffery's theory, i.e., in the absence of thermal noise. For $\mathrm{Pe} \gtrsim 1$, an excellent agreement is observed, indicating that the systematic tumbling motion dominates over the erratic Brownian motion. Lower Péclet numbers see a marked deviation from the theoretical period, with a drop in the average period. Notably, the Brownian motion consistently makes the particles perform a larger number of rotations than expected in a given time interval, instead of merely inducing fluctuations around the expected number of rotations. This suggests that the flow field biases the Brownian motion by acting as a ratchet that promotes

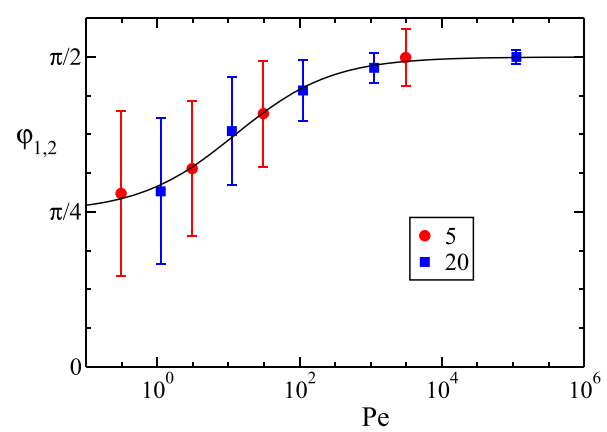

FIG. 5. The averaged location (markers) and standard deviations (error bars), plotted against the Péclet number, of the peaks in the orientation distribution functions of prolate spheroids ( $p=5$ and 20) subject to shear and Brownian motion, as determined by fitting distributions like those in Fig. 4 with the double-peaked function of Eq. (23). The line is a guide to the eye. 


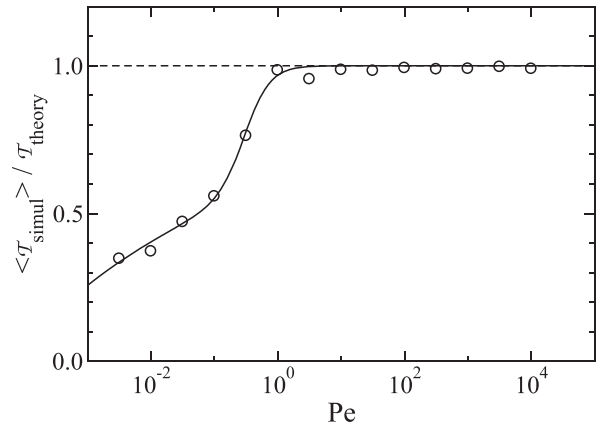

FIG. 6. The ratio of the average tumbling period in simulations to the theoretical tumbling period in the absence of noise, plotted against the Péclet number. The orientation of the spheroid, with the aspect ratio $p=5$, is constrained to the flow plane, $\theta=\pi / 2$.

rotation in the forward direction and hinders rotation in the reverse direction.

\section{Viscosity}

The instantaneous intrinsic viscosity in a non-Brownian simulation at constant $\theta=\pi / 2$ is shown in Fig. 7 to be in good agreement with Jeffery's expression, Eq. (18). The shear stress is at its lowest when the particle is slowly rotating through a flow-aligned state, $\varphi=(n+1 / 2) \pi$ with integer $n$, resulting in the two broad minima in the figure. Orientations away from the flow-aligned state tend to obstruct the flow and thereby increase the intrinsic viscosity. The maximum resistance, however, does not occur at the gradientaligned state, $\varphi=n \pi$, but at about $\varphi=(n \pm 1 / 4) \pi$, because the colloid traverses the gradient-aligned state with an angular velocity peaking at the flow field's shear rate, $\omega=\dot{\gamma}$, thereby briefly matching the flow field and hence giving rise to the narrow minima at $\varphi=n \pi$. At all of these minima, the intrinsic viscosity drops slightly below the constant value of $5 / 2$ for a sphere.

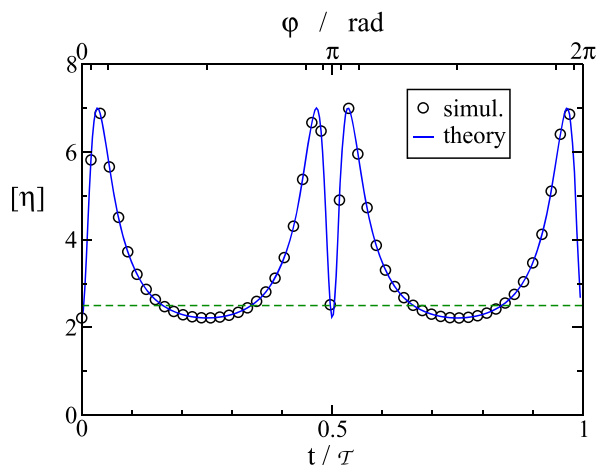

FIG. 7. The instantaneous intrinsic viscosity of a non-Brownian prolate spheroid $(p=5)$ tumbling in a simple shear flow at constant $\theta=\pi / 2$, as functions of time (bottom axis) and rotation angle (top axis, with ticks at intervals of $\Delta \varphi=\pi / 6$ ). The simulation results (markers) are in good agreement with Jeffery's theory; see Eqs. (17) and (18). The dashed line at $[\eta]=5 / 2$ highlights the intrinsic viscosity of a sphere.
Simulation results for the intrinsic viscosities of Brownian spheroidal colloids, over wide ranges of aspect ratios and Péclet numbers, are collected in Fig. 8 . Both prolate $(p>1)$ and oblate $(p<1)$ colloids show pronounced shear-thinning, which increases with the deviation from the spherical shape $(p=1)$. The decrease of the intrinsic viscosity with increasing Pe reflects the flow-induced alignment of the particles (see Figs. 4 and 5), which results in a reduction of the hindrance to the flow by the colloids; see Fig. 7 . With increasing Péclet number, shear thinning starts at $\mathrm{Pe} \sim 1$, irrespective of the aspect ratio, and finishes at $\mathrm{Pe} \sim p^{3}$ (prolate) or $\mathrm{Pe} \sim p^{-3}$ (oblate). The plateau values at a low Péclet, $[\eta]_{0}$, are plotted in Fig. 9 (top) as a function of the aspect ratio. A clear minimum of $[\eta]_{0}=5 / 2$ is reached for spherical colloids, with $[\eta]_{0}$ showing power law behavior for both smaller and larger aspect ratios. The solid lines represent the theoretical predictions of Eq. (22), which are in excellent agreement with the simulations for $p \leq 1 / 10$ and $p \geq 10$. The numerical data closely follow Simha's theory ${ }^{12}$ over the entire range of $p$. The plateau values of the intrinsic viscosities at a high Péclet, $[\eta]_{\infty}$, are plotted in Fig. 9 (bottom) as a function of the aspect ratio. For oblate spheroids, a plateau value of about 2.5 is obtained from the fits to the shear-thinning curves (see below), while the simulations at the highest Pe indicate a slightly higher plateau value of about 3 ; it should be noted that sampling configuration space by diffusion of the orbit parameter $C$ becomes prohibitively slow for extreme aspect ratios and high Péclet numbers. After a shallow minimum of $[\eta]_{\infty}=5 / 2$ for spheres, a power law growth of $[\eta]_{\infty}$ with $p$ sets in. The solid lines in the figure represent the theoretical predictions by Leal and Hinch ${ }^{13}$ [see Eq. (20)], which are in good

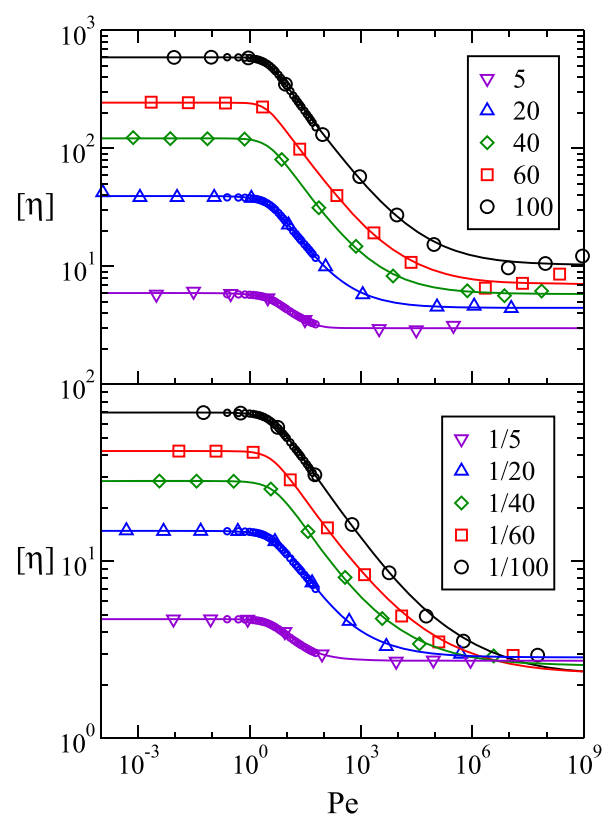

FIG. 8. The intrinsic viscosities of dilute suspensions of prolate (top) and oblate (bottom) spheroids of various aspect ratios $p$, see legends, as functions of the Péclet number. The smooth lines are fits with Eq. (25), yielding the fit parameters plotted in Figs. 9 and 10. The small markers for $0.25 \leq P e \leq 60$ represent numerical results by Scheraga. 


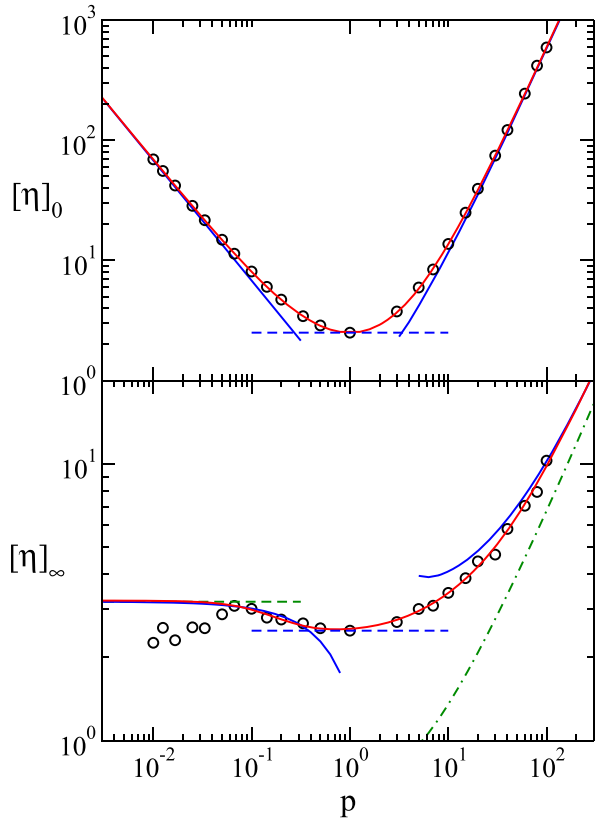

FIG. 9. The plateau values of the intrinsic viscosities $[\eta]$ in the low (top) and high (bottom) Péclet limits, obtained by fitting the data in Fig. 8 with the Carreau-like function of Eq. (25), plotted as functions of the aspect ratio $p$. The theoretical predictions of Eqs. (22) and (20) are included as solid blue lines in both plots and those of Eq. (21) as dashed-dotted green lines in the bottom plot. The red line in the top plot represents Simha's theory, ${ }^{12}$ and that in the bottom plot is a fit discussed in the main text. The dashed blue lines represent Einstein's result for a sphere, $[\eta]=5 / 2$.

agreement with the simulations for $p \leq 1 / 10$ and $p \geq 10$. The dashed lines denote their asymptotic predictions, Eq. (21), which are a good approximation for oblate spheroids at the smallest aspect ratios but for the prolate spheroid require aspect ratios much larger than those considered here. A nice fit covering the entire range is obtained with

$$
[\eta]_{\infty}=2.36+\frac{0.85+(1.58 p)^{2.60}}{1+(5.87 p)^{1.75}}
$$

where the less reliable data points for low $p$ were ignored in the fit.

The intrinsic viscosities in Fig. 8 are fitted very well with a variation on the Carreau model,

$$
[\eta]=\frac{[\eta]_{0}-[\eta]_{\infty}}{\left[1+(\alpha \mathrm{Pe})^{\beta}\right]^{\gamma}}+[\eta]_{\infty},
$$

drawn as solid lines in that figure. The two fit parameters for the plateau heights, $[\eta]_{0}$ and $[\eta]_{\infty}$, were discussed before. The fitted values for the scaling factor $\alpha$ and the powers $\beta$ and $\gamma$ are presented in Fig. 10. For the former, a V-shaped profile is observed, with

$$
\alpha=\left\{\begin{array}{rl}
-0.057 \ln p & \text { for } p<1 \\
0.085 \ln p & \text { for } p>1
\end{array},\right.
$$

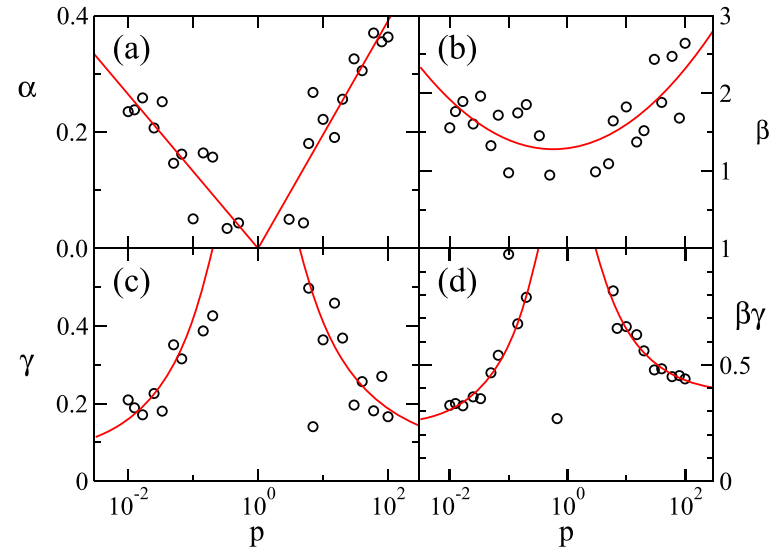

FIG. 10. Parametrization of the Carreau-like function [see Eq. (25)] fitted to the simulation data of Fig. 8. Shown are the variations with the aspect ratio of (a) the scaling factor $\alpha$, (b) the power $\beta$, (c) the power $\gamma$, and (d) the product $\beta \gamma$. The trend lines are discussed in the main text.

where the tip of $\alpha=0$ coincides with the absence of shear-thinning for a sphere; see Fig. 10(a). The outliers in the intermediate region around $p=1$ are probably a consequence of the small number of data points in the narrow shear-thinning regions for these spheroids and affect all four data sets in Fig. 10. The power $\beta$ is reasonably fitted with

$$
\beta=1.290+0.043 \ln p+0.039 \ln ^{2} p,
$$

plotted as a line in Fig. 10(b). The product of the powers $\beta$ and $\gamma$ is very well fitted by

$$
\beta \gamma=\left\{\begin{array}{lll}
0.23+1.63 p^{+0.66} & \text { for } & p<1 \\
0.37+1.30 p^{-0.66} & \text { for } & p>1
\end{array}\right.
$$

[see Fig. 10(d)]. Instead of fitting $\gamma$ independently, the ratio of the fits for $\beta \gamma$ and $\beta$ is shown in Fig. 10(c) to approximate $\gamma$ quite well. Of the three four-parameter variations on Eq. (25), both the Carreau model $(\beta=2)$ and the "hemi-Carreau" model $(\beta=1)$ perform considerably better than the Cross model $(\gamma=1)$, especially in the sharp corner at the onset of shear-thinning, with the Carreau and hemi-Carreau models working best for prolate and oblate spheroids, respectively.

It is suggested in the literature that the intrinsic viscosity in the shear-thinning region follows a power law, $[\eta] \propto \mathrm{Pe}^{-\delta}$, with $\delta=1 / 3$. ${ }^{5,11,72,75,76}$ Plots of the local powers $\delta(\mathrm{Pe})$ extracted from the simulations reveal that, following the onset of shear-thinning, $\delta$ rises within one to two orders in Pe to a maximum value that increases with $p$, barely reaching 0.3 for $p=1 / 100$ and just exceeding 0.4 for $p=100$, followed by a more gradual steady decay back to zero over many orders in Pe (data not shown). For strong shear thinning, the Carreau-like fit function reduces to a power law in the Péclet number, with $\delta=\beta \gamma$. The two aforementioned peak values of the local $\delta$ for the spheroids with the highest and lowest aspect ratios do indeed closely approximate the values of $\beta \gamma$ at these two extremes; see Fig. 10(d). Extrapolation based on the fit functions to $\beta \gamma$ then predicts the limit values of $\delta=0.37$ for prolate spheroids and $\delta=0.23$ for oblate spheroids. 
Figure 8 also shows data from the early electronic calculation by Scheraga, ${ }^{74}$ based on the theory by Saitô, ${ }^{77}$ combining a very slowly converging series of spherical harmonics for the orientation distribution function for near-spheres, due to Peterlin, ${ }^{78}$ with Jeffery's solution of the hydrodynamics for a spheroid. The numerical results, only available up to $\mathrm{Pe}=60$, are in excellent agreement with the intrinsic viscosities from our simulations-for the dozen $(\mathrm{Pe}, p)$ combinations where both values are available - and with the fitted functions to the latter.

\section{B. Complex particle shapes}

The methodology applied above to spheroids can also be used to colloids of more complex shapes. Whereas the generalized mobility matrix $\mathbf{M}$ of a sphere is block diagonal, a spheroid adds rotation-strain coupling, $\mathbf{M}_{E}^{\omega}$ and $\mathbf{M}_{\tau}^{S}$, a hemispherical cap adds translation-strain coupling, $\mathbf{M}_{E}^{v}$ and $\mathbf{M}_{f}^{S}$, and a helix adds rotationtranslation coupling, $\mathbf{M}_{\tau}^{v}$ and $\mathbf{M}_{f}^{\omega}$, thus step by step filling in all nine blocks of the matrix. Analytic expressions for the generalized mobility matrices of spheres and spheroids can be found in textbooks; those for the hemispherical cap and the helix were determined numerically by considering the bodies as rigid collections of nearly-touching unit-radius spherical primary particles, with interparticle hydrodynamic interactions calculated at the Rotne-PragerYamakawa level, ${ }^{79,80}$ using the publicly available 0 seen 11 code $^{61}$ as detailed in Ref. 42 and outlined in Appendix A. We verified that application of this technique to hollow spheroidal-shaped aggregates yields good agreement with the intrinsic viscosities discussed above.

\section{Hemispherical cap}

The hemispherical cap (see Fig. 1) was selected as the next step in complexity following the spheroid. Analytical expressions for the Jeffery orbit of a spherical cap in a linear shear flow were derived by Dorrepaal, ${ }^{47}$ showing that the cap combines the periodic tumbling motion of spheroids with simultaneous translations of the hydrodynamic center along both the velocity and velocity-gradient directions. As shown in our previous work, ${ }^{42}$ the employed simulation method accurately captures this motion (in the absence of Brownian noise). A cap-like rigid aggregate, with a top angle $\alpha=\pi / 2$, was constructed by positioning 2051 primary particles on the corner points of a triangulated mesh generated with the DistMesh routine ${ }^{81}$ in $\mathrm{MATLAB}^{82}{ }^{82}$ resulting in a cap with a radius $R \approx 47.8 \sigma$. The Péclet number is again defined as $\mathrm{Pe}=\dot{\gamma} / D_{r}$, where the rotational diffusion coefficient $D_{r}$ is obtained as $k_{B} T$ times the degenerate eigenvalue of $\mathbf{M}_{\tau}^{\omega}$. The colloidal volume is taken as the volume enclosed by the hemisphere, $v_{\mathrm{c}}=\frac{2}{3} \pi R^{3}$. Simulation results for the intrinsic viscosity as a function of Péclet number, as presented in Fig. 11, systematically exceed the sphere's $[\eta]=5 / 2$ by $10 \%-20 \%$. The hemispherical cap shows only weak shear-thinning because, at a length to width to height ratio of about $2: 2: 1$, this colloid is still fairly close to spherical in shape.

\section{Helix}

The final colloidal shape explored in this study is the helix, lacking any symmetry and requiring all nine blocks of the mobility matrix. Due to this complexity, there are no analytic solutions to

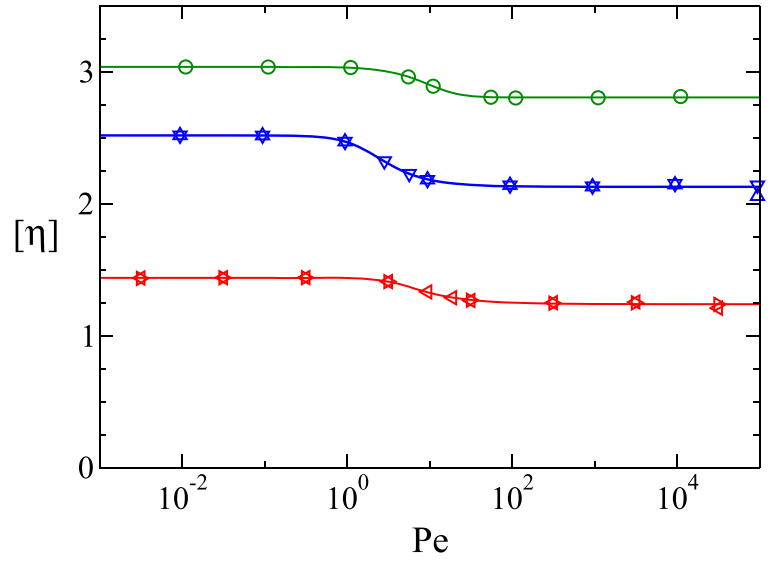

FIG. 11. The intrinsic viscosities of a hemispherical cap (green circles), of two short helices (red triangles pointing left and right for left and right-handedness, respectively), and of two long helices (blue triangles pointing up and down for left and right-handedness, respectively) as functions of the Péclet number. The lines are fit with Eq. (25).

its intricate motion under shear. We modeled helices as rigid collections of primary particles distributed along a spiral of diameter $D=63.3 \sigma$; see Fig. 1. A short helix of two turns comprised 201 particles, amounting to a length of $L=41.8 \sigma$, and a long helix of 7.5 turns and 751 particles reached a length of $L=158.3$. For both helices, left and right-handed versions were created.

In the absence of Brownian motion, the long helix periodically tumbles its long axis and rotates around this axis, like the spheroids, while the hydrodynamic center is moving periodically along both the velocity and velocity-gradient directions, like the cap. Unlike either of these two bodies, the helix also moves along the vorticity direction, combining a periodic back and forth motion with a steady drift. The right-handed helix with its long axis tumbling in the shear plane $\theta \approx \pi / 2$ drifts in the negative vorticity direction. Rotating the long axis to align with the vorticity direction, $\theta \approx 0$, flips the drift to the positive vorticity direction and reduces the (absolute) drift velocity by about two orders in magnitude. The latter drift reflects the usual motion of a screw, turning rotation around the axis into motion along the axis. The former sideward motion is caused by a lift force that varies and can change sign with the rotation angle $\varphi$ in the shear plane and the rotation angle of the helix around its long axis; even the (noninteger) number of turns of the helix can alter the direction of the lift force. ${ }^{50,52}$ Upon introducing Brownian motion, the righthanded helix shows an average drift along the negative vorticity direction. All drifts are reversed for a left-handed helix. The motion of the short helix is less predictable; even in the absence of Brownian noise, particles with their central axes initially aligned in or perpendicular to the shear flow do not maintain their orientations. ${ }^{48}$ The short helices were seen to drift along both the positive and the negative vorticity direction, and changes in the direction were seen as late as $10^{6} \tau$.

The intrinsic viscosities of the helices are presented in Fig. 11. The Péclet number is again defined as $\mathrm{Pe}=\dot{\gamma} / D_{r}$ where the rotational diffusion coefficient $D_{r}$ is now obtained as $k_{B} T$ times the value of $\mathbf{M}_{\tau}^{\omega}$ along the helix's long axis, and the volume is calculated as that 
of the circumscribing cylinder, $v_{\mathrm{c}}=\frac{1}{4} \pi D^{2} L$. Since all four helices share aspect ratios $p=L / D$ fairly close to unity, they show modest shear thinning. The low values of the intrinsic viscosities, relative to spheroids of similar aspect ratios, indicate that the impact of the helix on the surrounding and enclosed fluid is less than that of similarly sized spheroids. The handedness of the helix appears to play no role, as evidenced from the near coalescence of the markers in Fig. 11.

\section{SUMMARY AND CONCLUSIONS}

A recently proposed efficient Brownian dynamics scheme, utilizing quaternions for the rotational dynamics and taking advantage of the mobility matrix being constant in the body-based frame, has been used to simulate the dynamics of arbitrarily shaped colloids subjected simultaneously to simple shear and Brownian motions. The calculated intrinsic viscosities for spheroids are in excellent agreement with theoretical predictions in the limits of high and low Péclet values, providing support for the validity of both theory and the employed simulation methodology. Intrinsic viscosities at intermediate Péclet values, in the shear thinning regime, are well fitted with a modified Carreau model. Fit functions are presented for the model parameters, covering a wide range of aspect ratios, to provide a benchmark for future theories, simulations, and experiments. The applied methods work for particles of the arbitrary shape, as illustrated by the viscosity calculations for a cap and helices.

\section{ACKNOWLEDGMENTS}

This work was part of the "Computational Sciences for Energy Research" Industrial Partnership Programme, co-financed by Shell Global Solutions B.V. and the Netherlands Organization for Scientific Research (NWO). We thank Professor Stefan Luding for stimulating discussions.

\section{APPENDIX A: MOBILITY MATRIX}

This appendix summarizes the derivation of the generalized mobility matrix of a rigid cluster of $N$ identically sized spherical primary particles, as described in more detail in our previous work. ${ }^{42}$ In the mobility picture, the hydrodynamic interactions couple every particle $i$ with every particle $j$,

$$
\left(\begin{array}{c}
\overline{\mathbf{v}}_{i}-\overline{\mathbf{v}}_{\infty}\left(\overline{\mathbf{x}}_{i}\right) \\
\overline{\boldsymbol{\omega}}_{i}-\overline{\boldsymbol{\omega}}_{\infty} \\
-\overline{\overline{\mathbf{E}}}_{\infty}
\end{array}\right)=\sum_{j=i}^{N}\left(\begin{array}{ccc}
\overline{\overline{\mathbf{M}}}_{f, j}^{v, i} & \overline{\overline{\mathbf{M}}}_{\tau, j}^{v, i} & \overline{\overline{\mathbf{M}}}_{S, j}^{v, i} \\
\overline{\overline{\mathbf{M}}}_{f, j}^{\omega, i} & \overline{\overline{\mathbf{M}}}_{\tau, j}^{\omega, i} & \overline{\overline{\mathbf{M}}} \\
\overline{\overline{\mathbf{M}}}_{S, i} \\
\overline{\mathbf{M}}_{f, j} & \overline{\overline{\mathbf{M}}}_{\tau, j}^{E, i} & \overline{\bar{\Xi}}_{S, j}^{E, i}
\end{array}\right)\left(\begin{array}{c}
\overline{\mathbf{f}}_{j} \\
\overline{\boldsymbol{\tau}}_{j} \\
\overline{\overline{\mathbf{S}}}_{j}
\end{array}\right) .
$$

Expressions for the various pair mobility matrices can be found in the literature. ${ }^{53,54,83,84}$ The combination of variously sized matrices excludes the use of conventional rank two matrix manipulations; hence, it proves convenient to reduce the strain and stress matrices to vectors. In the current case, both the strain and the stress are symmetric and traceless, and hence can be expressed as five-vectors. The conversion of the strain is realized by rewriting the matrix as a linear combination of five $(3 \times 3)$ "basis matrices" $\overline{\mathbf{e}}_{\kappa}^{E}$,

$$
\begin{aligned}
& \overline{\overline{\mathbf{E}}}_{\infty}=\sum_{\kappa} \overline{\overline{\mathbf{e}}}_{\kappa}^{E} \mathcal{E}_{\infty}^{\kappa}, \\
& \mathcal{E}_{\infty}^{\kappa}=\overline{\overline{\mathbf{e}}}_{E}^{\kappa}: \overline{\overline{\mathbf{E}}}_{\infty},
\end{aligned}
$$

where the five parameters $\mathcal{E}_{\infty}^{\kappa}$ combine into the vector $\overline{\mathcal{E}}_{\infty}$ and the second line, with $\overline{\overline{\mathbf{e}}}_{E}^{\kappa}: \overline{\overline{\mathbf{e}}}_{\lambda}^{E}=\delta_{\lambda}^{\kappa}$ and $\delta$ the Kronecker-delta, provides the inverse transformation. A likewise conversion is used for the stress matrices $\overline{\mathbf{S}}_{j}$, yielding the vectors $\overline{\mathcal{S}}_{j}$. The basis matrices need not be orthogonal, but it is recommendable to select $\overline{\overline{\mathbf{e}}}_{\kappa}^{E}=\overline{\overline{\mathbf{e}}}_{S}^{\kappa}$ in order to retain a symmetric grand mobility matrix. After re-expressing the grand mobility in terms of the strain and stress vectors, it reduces to a regular rank-two matrix. Inversion by standard algorithms, which is equivalent to summing over all reflections between all particles, ${ }^{53}$ yields

$$
\left(\begin{array}{c}
\mathbf{f}_{i} \\
\boldsymbol{\tau}_{i} \\
\mathcal{S}_{i}
\end{array}\right)=\sum_{j=1}^{N}\left(\begin{array}{lll}
\mathbf{R}_{v, j}^{f, i} & \mathbf{R}_{\omega, j}^{f, i} & \mathbf{R}_{\mathcal{E}, j}^{f, i} \\
\mathbf{R}_{v, j}^{\tau, i} & \mathbf{R}_{\omega, j}^{\tau, i} & \mathbf{R}_{\mathcal{E}, j}^{\tau, i} \\
\mathbf{R}_{v, j}^{\mathcal{S}, i} & \mathbf{R}_{\omega, j}^{\mathcal{S}, i} & \mathbf{R}_{\mathcal{E}, j}^{\mathcal{S}, i}
\end{array}\right)\left(\begin{array}{c}
\mathbf{v}_{j}-\mathbf{v}_{\infty}\left(\mathbf{x}_{j}\right) \\
\boldsymbol{\omega}_{j}-\boldsymbol{\omega}_{\infty} \\
-\mathcal{E}_{\infty}
\end{array}\right),
$$

where the bars have been omitted. In the vector on the RHS, the velocities of and at the particles are related to the velocities of and at the cluster's center via the rigidity of the cluster and the linearity of the flow,

$$
\begin{aligned}
\mathbf{v}_{j} & =\mathbf{v}+\boldsymbol{\omega} \times \mathbf{r}_{j}, \\
\boldsymbol{\omega}_{j} & =\boldsymbol{\omega}, \\
\mathbf{v}_{\infty}\left(\mathbf{x}_{j}\right) & =\mathbf{v}_{\infty}(\mathbf{x})+\boldsymbol{\omega}_{\infty} \times \mathbf{r}_{j}+\mathbf{E}^{\infty} \mathbf{r}_{j},
\end{aligned}
$$

where $\mathbf{r}_{j}=\mathbf{x}_{j}-\mathbf{x}$. In the vector on the LHS of Eq. (A3), the forces, torques, and stresses on the particles can be summed into the total force, torque, and stress on the cluster,

$$
\begin{aligned}
& \mathbf{f}=\sum_{i=1}^{N} \mathbf{f}_{i}, \\
& \boldsymbol{\tau}=\sum_{i=1}^{N}\left(\boldsymbol{\tau}_{i}+\mathbf{r}_{i} \times \mathbf{f}_{i}\right), \\
& \mathbf{S}=\sum_{i=1}^{N}\left(\mathbf{S}_{i}+\mathbf{r}_{i} \otimes \mathbf{f}_{i}\right) .
\end{aligned}
$$

By combining the above steps, ${ }^{42}$ one arrives at the grand resistance matrix of the cluster,

$$
\left(\begin{array}{c}
\mathbf{f} \\
\boldsymbol{\tau} \\
\mathcal{S}
\end{array}\right)=\left(\begin{array}{lll}
\mathbf{R}_{v}^{f} & \mathbf{R}_{\omega}^{f} & \mathbf{R}_{\mathcal{E}}^{f} \\
\mathbf{R}_{v}^{\tau} & \mathbf{R}_{\omega}^{\tau} & \mathbf{R}_{\mathcal{E}}^{\tau} \\
\mathbf{R}_{v}^{\mathcal{S}} & \mathbf{R}_{\omega}^{\mathcal{S}} & \mathbf{R}_{\mathcal{E}}^{\mathcal{S}}
\end{array}\right)\left(\begin{array}{c}
\mathbf{v}-\mathbf{v}_{\infty}(\mathbf{x}) \\
\boldsymbol{\omega}-\boldsymbol{\omega}_{\infty} \\
-\mathcal{E}_{\infty}
\end{array}\right)
$$

A partial inversion of the above expression, bringing the velocities to the left and the forces to the right, followed by a conversion back to stress and strain matrices, yields Eq. (2). Note that this partial inversion implies that the $(5 \times 6)$ block $\mathbf{M}_{F}^{\mathcal{S}}$ of the Cartesian mobility matrix is related to the $(5 \times 6)$ block $\mathbf{R}_{\dot{Q}}^{\mathcal{S}}$ and the $(6 \times 6)$ block $\mathbf{R}_{\dot{Q}}^{F}$ of the corresponding resistance matrix by

$$
\mathbf{M}_{F}^{\mathcal{S}}=\mathbf{R}_{\dot{Q}}^{\mathcal{S}}\left(\mathbf{R}_{\dot{Q}}^{F}\right)^{-1} .
$$


The stress-term $\mathbf{G}^{(\mathrm{s})}$, as derived by Bossis and Brady ${ }^{71}$ in the resistance picture, is then readily converted to the mobility form used in Eq. (11).

\section{APPENDIX B: QUATERNIONS}

Unit quaternions, ${ }^{41}$ i.e., a four-vector $\mathbf{q}=\left(q_{0}, q_{1}, q_{2}, q_{3}\right)$ of unit length $q=|\mathbf{q}|=1$, provide a convenient way to parameterize a rotation matrix in three-dimensional space. The rotation matrix for the conversion from body-based to space-based coordinates reads as

$$
\mathbf{A}_{(\mathrm{b})}^{(\mathrm{s})}=\left(\begin{array}{lcr}
q_{0}^{2}+q_{1}^{2}-q_{2}^{2}-q_{3}^{2} & 2 q_{1} q_{2}-2 q_{0} q_{3} & 2 q_{1} q_{3}+2 q_{0} q_{2} \\
2 q_{1} q_{2}+2 q_{0} q_{3} & q_{0}^{2}-q_{1}^{2}+q_{2}^{2}-q_{3}^{2} & 2 q_{2} q_{3}-2 q_{0} q_{1} \\
2 q_{1} q_{3}-2 q_{0} q_{2} & 2 q_{2} q_{3}+2 q_{0} q_{1} & q_{0}^{2}-q_{1}^{2}-q_{2}^{2}+q_{3}^{2}
\end{array}\right),
$$

while the inverse conversion $\mathbf{A}_{(\mathrm{s})}^{(\mathrm{b})}$ is realized by the transpose of $\mathbf{A}_{(\mathrm{b})}^{(\mathrm{s})}$. A conversion matrix between angular velocities in the space frame and quaternion velocities is obtained by evaluating $\omega^{(s)} \times \mathbf{x}^{(s)}$ $=\dot{\mathbf{A}}_{(\mathrm{b})}^{(\mathrm{s})} \mathbf{A}_{(\mathrm{s})}^{(\mathrm{b})} \mathbf{x}^{(\mathrm{s})}$, which yields

$$
\mathbf{B}_{\omega(\mathrm{s})}^{\dot{q}}=\frac{\partial \dot{\mathbf{q}}}{\partial \boldsymbol{\omega}^{(s)}}=\frac{1}{2 q^{4}}\left(\begin{array}{ccc}
-q_{1} & -q_{2} & -q_{3} \\
q_{0} & q_{3} & -q_{2} \\
-q_{3} & q_{0} & q_{1} \\
q_{2} & -q_{1} & q_{0}
\end{array}\right) .
$$

The conversion from an angular velocity in the body frame to a quaternion velocity can be derived likewise, yielding

$$
\mathbf{B}_{\omega(\mathrm{b})}^{\dot{q}}=\frac{\partial \dot{\mathbf{q}}}{\partial \boldsymbol{\omega}^{(\mathrm{b})}}=\frac{1}{2 q^{4}}\left(\begin{array}{lrr}
-q_{1} & -q_{2} & -q_{3} \\
q_{0} & -q_{3} & q_{2} \\
q_{3} & q_{0} & -q_{1} \\
-q_{2} & q_{1} & q_{0}
\end{array}\right),
$$

and can also be obtained from $\mathbf{B}_{\omega(\mathrm{b})}^{\dot{q}}=\mathbf{B}_{\omega(\mathrm{s})}^{\dot{q}} \mathbf{A}_{(\mathrm{b})}^{(\mathrm{s})}$.

\section{APPENDIX C: ROTATIONAL DERIVATIVES}

The derivative of a function $f(\mathbf{r})$ under a rotation of the point $\mathbf{r}$ around the coordinate axis $\hat{\mathbf{e}}_{\alpha}$ through the origin reads as

$$
\mathcal{R}_{\alpha} f(\mathbf{r})=\frac{\partial f(\mathbf{r})}{\partial \mathbf{r}} \cdot\left(\hat{\mathbf{e}}_{\alpha} \times \mathbf{r}\right)=\epsilon_{\alpha \beta}^{\gamma} r^{\beta} \frac{\partial f(\mathbf{r})}{\partial r^{\gamma}},
$$

where the Levi-Civita symbol $\epsilon_{\beta \gamma}^{\alpha}$ returns $+1(-1)$ if $\{\alpha, \beta, \gamma\}$ is a cyclic (anticyclic) combination of $\{x, y, z\}$, and zero otherwise. A right-handed rotation matrix $\mathbf{A}$ can be regarded as a collection of three perpendicular unit column vectors, $\mathbf{A}=\left(\mathbf{a}_{1} \mathbf{a}_{2} \mathbf{a}_{3}\right)$, with the $(\lambda, \kappa)$ th matrix element equaling the $\lambda$ th element of the $\kappa$ th vector, $A_{\kappa}^{\lambda}=\left(a_{\kappa}\right)^{\lambda}$. Defining a function $f(\mathbf{r})=\mathbf{r} \cdot \hat{\mathbf{e}}_{\lambda}=r^{\lambda}$, one readily shows that

$$
\mathcal{R}_{\alpha} A_{\kappa}^{\lambda}=\mathcal{R}_{\alpha} f\left(\mathbf{a}_{\kappa}\right)=\epsilon_{\alpha \beta}^{y}\left(a_{\kappa}\right)^{\beta} \frac{\partial\left(a_{\kappa}\right)^{\lambda}}{\partial\left(a_{\kappa}\right)^{\gamma}}=\epsilon_{\alpha \beta}^{\lambda} A_{\kappa}^{\beta},
$$

which is used in Eq. (12). Taking the cross product of two column vectors of a rotation matrix gives

$$
\epsilon_{\beta \gamma}^{\alpha} A_{\kappa}^{\beta} A_{\lambda}^{\gamma}=\left(\mathbf{a}_{\kappa} \times \mathbf{a}_{\lambda}\right)_{\alpha}=\left(\epsilon_{\kappa \lambda}^{\mu} \mathbf{a}_{\mu}\right)_{\alpha}=\epsilon_{\kappa \lambda}^{\mu} A_{\mu}^{\alpha},
$$

where the right-handedness of $\mathbf{A}$ was used in the second step.

\section{REFERENCES}

${ }^{1}$ A. Einstein, Ann. Phys. 324, 289 (1906).

${ }^{2}$ A. Einstein, Ann. Phys. 339, 591 (1911).

${ }^{3}$ G. B. Jeffery, Proc. R. Soc. A 102, 161 (1922).

${ }^{4}$ F. P. Bretherton, J. Fluid Mech. 14, 284 (1962).

${ }^{5}$ H. Brenner, Int. J. Multiphase Flow 1, 195 (1974).

${ }^{6}$ V. Dabade, N. K. Marath, and G. Subramanian, J. Fluid Mech. 791, 631 (2016).

${ }^{7} \mathrm{~J}$. M. Burgers, "On the motion of small particles of elongated form suspended in a viscous liquid," in Second Report on Viscosity and Plasticity (Noord-Hollandsche Uitgeversmaatschappij, Amsterdam, The Netherlands, 1938), Chap. III, reprinted in F. T. Nieuwstadt and J. A. Steketee, Selected Papers of J. M. Burgers (Springer, Dordrecht, The Netherlands, 1995).

${ }^{8}$ L. Onsager, Phys. Rev. 40, 1028 (1932).

${ }^{9}$ W. Kuhn and H. Kuhn, Helv. Chim. Acta 28, 97 (1945).

${ }^{10}$ H. Giesekus, Rheol. Acta 2, 50 (1962).

${ }^{11}$ E. J. Hinch and L. G. Leal, J. Fluid Mech. 52, 683 (1972).

${ }^{12}$ R. Simha, J. Phys. Chem. 44, 25 (1940).

${ }^{13}$ L. G. Leal and E. J. Hinch, J. Fluid Mech. 46, 685 (1971).

${ }^{14}$ R. Rutgers, Rheol. Acta 2, 202 (1962).

${ }^{15}$ R. Rutgers, Rheol. Acta 2, 305 (1962).

${ }^{16}$ A. M. Wierenga and A. P. Philipse, Colloids Surf., A 137, 355 (1998).

${ }^{17}$ S. Mueller, E. W. Llewellin, and H. M. Mader, Proc. R. Soc. A 466, 1201 (2010).

${ }^{18}$ B. J. Konijn, O. B. J. Sanderink, and N. P. Kruyt, Powder Tech. 266, 61 (2014).

${ }^{19}$ F. Lundell and A. Carlsson, Phys. Rev. E 81, 016323 (2010).

${ }^{20}$ J. Einarsson, J. R. Angilella, and B. Mehlig, Physica D 278-279, 79 (2014).

${ }^{21}$ Z. Yu, N. Phan-Thien, and R. I. Tanner, Phys. Rev. E 76, 026310 (2007).

${ }^{22}$ H. Huang, X. Yang, M. Krafczyk, and X.-Y. Lu, J. Fluid Mech. 692, 369 (2012).

${ }^{23}$ W. Mao and A. Alexeev, J. Fluid Mech. 749, 145 (2014).

${ }^{24}$ M. Daghooghi and I. Borazjani, J. Fluid Mech. 781, 506 (2015).

${ }^{25}$ T. Rosén, Chaos 27, 063112 (2017).

${ }^{26}$ G. Almondo, J. Einarsson, J. R. Angilella, and B. Mehlig, Phys. Rev. Fluids 3, 064307 (2018).

${ }^{27}$ N. K. Marath, R. Dwivedi, and G. Subramanian, J. Fluid Mech. 811, R3 (2017).

${ }^{28}$ M. Makino and M. Doi, J. Phys. Soc. Jpn. 73, 2739 (2004).

${ }^{29}$ Y. M. Harshe, L. Ehrl, and M. Lattuada, J. Colloid Interface Sci. 352, 87 (2010).

${ }^{30}$ J. Wang, E. J. Tozzi, M. D. Graham, and D. J. Klingenberg, Phys. Fluids 24, 123304 (2012).

${ }^{31}$ M. Daghooghi and I. Borazjani, J. Fluid Mech. 839, 663 (2018).

${ }^{32}$ T. Rosén, F. Lundell, and C. Aidun, J. Fluid Mech. 738, 563 (2014).

${ }^{33}$ B. Leahy, D. L. Koch, and I. Cohen, J. Fluid Mech. 772, 42 (2015).

${ }^{34}$ M. Makino and M. Doi, Phys. Fluids 17, 103605 (2005).

${ }^{35}$ M. Makino and M. Doi, J. Phys. Soc. Jpn. 73, 3020 (2004).

${ }^{36}$ Y. M. Harshe and M. Lattuada, J. Colloid Interface Sci. 367, 83 (2012).

${ }^{37} \mathrm{BEST}$ is available upon request from aragons@sfsu.edu.

${ }^{38}$ See http://leonardo.inf.um.es/macromol/programs/hydro++/hydro++.htm for HYDRO++.

${ }^{39}$ D. K. Hahn and S. R. Aragon, J. Chem. Theo. Comput. 2, 1416 (2006).

${ }^{40}$ J. García de la Torre, G. del Rio Echenique, and A. Ortega, J. Phys. Chem. B 111, 955 (2007).

${ }^{41}$ H. Goldstein, Classical Mechanics, 2nd ed. (Addison-Wesley, Reading, MA, USA, 1980).

${ }^{42}$ D. Palanisamy and W. K. den Otter, J. Chem. Phys. 148, 194112 (2018).

${ }^{43}$ E. Dickinson, Chem. Soc. Rev. 14, 421 (1985). 
${ }^{44}$ R. Kutteh, J. Chem. Phys. 132, 174107 (2010)

${ }^{45}$ I. Torres-Díaz and C. Rinaldi, J. Phys. D 47, 235003 (2014).

${ }^{46}$ I. M. Ilie, W. J. Briels, and W. K. den Otter, J. Chem. Phys. 142, 114103 (2015).

${ }^{47}$ J. M. Dorrepaal, J. Fluid Mech. 84, 265 (1978).

${ }^{48}$ Y.-J. Kim and W. J. Rae, Int. J. Multiphase Flow 17, 717 (1991).

${ }^{49}$ P. Chen and C.-H. Chao, Phys. Fluids 19, 017108 (2007).

${ }^{50}$ Marcos, H. C. Fu, T. R. Powers, and R. Stocker, Phys. Rev. Lett. 102, 158103 (2009).

${ }^{51}$ S. Ro, J. Yi, and Y. W. Kim, Sci. Rep. 6, 35144 (2016).

${ }^{52}$ Q.-Y. Zhang, K.-Y. Hu, and W.-Y. Yang, Mod. Phys. Lett. B 31, 1750117 (2017).

${ }^{53}$ L. Durlofsky, J. F. Brady, and G. Bossis, J. Fluid Mech. 180, 21 (1987).

${ }^{54}$ S. Kim and S. Karrila, Microhydrodynamics: Principles and Selected Applications, Butterworth-Heinemann Series in Chemical Engineering (ButterworthHeinemann, Stoneham, MA, USA, 1991).

${ }^{55}$ E. Guazzelli and J. F. Morris, A Physical Introduction to Suspension Dynamics, Cambridge Texts in Applied Mathematics (Cambridge University Press, Cambridge, UK, 2012).

${ }^{56}$ S. Aragon, J. Comput. Chem. 25, 1191 (2004).

${ }^{57}$ S. Aragon, Methods 54, 101 (2011).

${ }^{58}$ B. Carrasco and J. García de la Torre, J. Chem. Phys. 111, 4817 (1999).

${ }^{59}$ R. Seto, R. Botet, and H. Briesen, Phys. Rev. E 84, 041405 (2011).

${ }^{60}$ J. J. Molina and R. Yamamoto, J. Chem. Phys. 139, 234105 (2013).

${ }^{61}$ See https://www2.msm.ctw.utwente.nl/Oseen11/ for Oseen11.

${ }^{62}$ N. G. van Kampen, Stochastic Processes in Physics and Chemistry, Revised and Enlarged Edition (Elsevier, Amsterdam, The Netherlands, 1992).

${ }^{63}$ H. C. Öttinger, Stochastic Processes in Polymeric Fluids (Springer-Verlag, Berlin, Germany, 1996).
${ }^{64}$ C. Gardiner, Stochastic Methods: A Handbook for the Natural and Social Sciences, Springer Series in Synergetics, 4th ed. (Springer-Verlag, Berlin, Germany, 2009).

${ }^{65}$ S. N. Naess and A. Elgsaeter, Macromol. Theory Simul. 13, 419 (2004).

${ }^{66}$ T. R. Evensen, A. Elgsaeter, and S. N. Naess, Colloids Surf., B 56, 80 (2007).

${ }^{67}$ T. R. Evensen, S. N. Naess, and A. Elgsaeter, Macromol. Theory Simul. 17, 121 (2008).

${ }^{68}$ T. R. Evensen, S. N. Naess, and A. Elgsaeter, Macromol. Theory Simul. 17, 403 (2008).

${ }^{69}$ T. R. Evensen, S. N. Naess, and A. Elgsaeter, Macromol. Theory Simul. 18, 50 (2009).

${ }^{70}$ G. K. Batchelor, J. Fluid Mech. 83, 97 (1977).

${ }^{71}$ G. Bossis and J. F. Brady, J. Chem. Phys. 91, 1866 (1989).

${ }^{72}$ M. Doi and S. F. Edwards, The Theory of Polymer Dynamics, International Series of Monographs in Physics (Oxford Science Publishers, Oxford, UK, 2013), Vol. 73.

${ }^{73}$ J. K. G. Dhont, An Introduction to Dynamics of Colloids, Studies in Interface Science (Elsevier, Amsterdam, The Netherlands, 1996).

${ }^{74}$ H. A. Scheraga, J. Chem. Phys. 23, 1526 (1955).

${ }^{75}$ W. E. Stewart and J. P. Sorensen, Trans. Soc. Rheol. 16, 1 (1972).

${ }^{76} \mathrm{P}$. Oswald, Rheophysics: The Deformation of Flow and Matter (Cambridge University Press, Cambridge, UK, 2009).

${ }^{77}$ N. Saitô, J. Phys. Soc. Jpn. 6, 297 (1951).

${ }^{78}$ A. Peterlin, Z. Phys. 111, 232 (1938).

${ }^{79}$ J. Rotne and S. Prager, J. Chem. Phys. 50, 4831 (1969).

${ }^{80}$ H. Yamakawa, J. Chem. Phys. 53, 436 (1970).

${ }^{81}$ P.-O. Persson and G. Strang, SIAM Rev. 46, 329 (2004).

${ }^{82}$ MATLAB R2014a, The MathWorks, Inc., Natick, MA, USA, 2014.

${ }^{83}$ D. J. Jeffrey and Y. Onishi, J. Fluid Mech. 139, 261 (1984).

${ }^{84}$ S. Kim and R. T. Mifflin, Phys. Fluids 28, 2033 (1985). 